\title{
Motor nuclei innervating eye muscles spared in mouse model of SOD1-linked ALS
}

Eleanor V. Thomas ${ }^{1}$, Maria Nagy ${ }^{2,3}$, Hongyu Zhao 2,4 , Wayne A. Fenton ${ }^{2}$, and

Arthur L. Horwich ${ }^{2,3}$

${ }^{1}$ Interdepartmental Program in Neuroscience, Yale University School of Medicine, New Haven, CT 06510

2Dept Genetics, Yale University School of Medicine, New Haven, CT 06510

${ }^{3}$ Howard Hughes Medical Institute

${ }^{4}$ Dept. Biostatistics, Yale University School of Public Health, New Haven, CT 06510

Correspondence: arthur.horwich@yale.edu 


\section{Summary.}

The eye muscles of humans with either inherited or sporadic forms of ALS are relatively unaffected during disease progression, a function of sparing of the cranial nerve motor neurons supplying them. Here we observe that cranial nerve nuclei are also spared in a mouse model of inherited SOD1-linked ALS. We examined the cranial nerve motor nuclei in a mouse strain, G85R SOD1YFP, which carries a high copy transgene encoding a mutant human SOD1-YFP fusion protein, that exhibits florid YFP-fluorescent aggregates in spinal cord motor neurons and paralyzes by 6 months of age. We observed in the cranial nerve nuclei that innervate the eye, $3 \mathrm{~N}$ (oculomotor), $4 \mathrm{~N}$ (trochlear), and $6 \mathrm{~N}$ (abducens), that there was little $(4 \mathrm{~N}, 6 \mathrm{~N})$ or no $(3 \mathrm{~N})$ aggregation, in comparison with other motor nuclei, $5 \mathrm{~N}$ (trigeminal), $7 \mathrm{~N}$ (facial), and 12N (hypoglossal), in the latter two of which florid aggregation was observed. Correspondingly, the number of ChAT positive motor neurons in $3 \mathrm{~N}$ of G85R SOD1YFP relative to that in $3 \mathrm{~N}$ of ChAT-EGFP mice showed that there was no loss of motor neurons over time, whereas in $12 \mathrm{~N}$ there was progressive loss of motor neurons, amounting to a loss of $\sim 30 \%$ from G85R SOD1YFP by end-stage. Thus, the sparing of extraocular motor neurons as occurs in humans with ALS appears to be replicated in our SOD1-linked ALS mouse strain, supporting the validity of the mouse model for studying this aspect of selective motor system loss in ALS. Comparisons of extraocular motor neurons (e.g., from $3 \mathrm{~N}$ ), resistant to ALS pathology, with other cranial motor neurons (e.g., from $12 \mathrm{~N}$ ), sensitive to such pathology, may thus be of value in understanding mechanisms of protection vs. susceptibility of motor neurons. 
The sparing of eye movements in ALS patients is well-known and has been related to lack of involvement of the motor nuclei innervating the extraocular muscles, namely, cranial nerve nuclei 3N (oculomotor), 4N (trochlear), and 6N (abducens) (ref. 1 and references therein). We asked whether our mouse model of ALS, bearing a high copy number transgene of a human genomic clone of G85R mutant SOD1 (including the human promoter) fused in its last exon with YFP (2,3), might likewise exhibit sparing of the same cranial nerve motor nuclei as compared with the other cranial motor nuclei. Notably, spinal cord motor neurons in our mouse strain exhibit large YFP fluorescent aggregates by 1 month of age, which increase in number subsequently and are associated with motor neuron cell death, amounting to $50 \%$ loss by 3 months of age and $75 \%$ loss by end-stage (3). The mice exhibit lower extremity symptoms by 3 months of age - twitching or pulling in when picked up by the tail - and uniformly paralyze by 6 months of age (4). We thus examined the cranial nerve nuclei at these two times, in each case examining 3 mice. At both 3 months and 6 months (end-stage), we observed no aggregation in ChAT positive motor neurons in $3 \mathrm{~N}$ (see representative images in Fig.1A and 1B, and quantitation in bar graphs below and Table 1). In particular, 3N exhibited no aggregates in greater than 500 ChAT-positive motor neurons examined, 200 examined from three mice at 3 months and $\sim 300$ examined from three mice at end-stage. The two other extraocular nuclei, $4 \mathrm{~N}$ and $6 \mathrm{~N}$, exhibited rare aggregates at 3 months of age and essentially none at end-stage (see Fig. 1 bar graphs and Table 1). By contrast, $7 \mathrm{~N}$ and $12 \mathrm{~N}$ exhibited florid aggregation at both times (Fig. 1A, 1B, and Table 1), affecting $\sim 20-30 \%$ of neurons, whereas $5 \mathrm{~N}$ was less affected, exhibiting a level of aggregation resembling that of $4 \mathrm{~N}$ and $6 \mathrm{~N}$ at 3 months, but greater than $3 \mathrm{~N}, 4 \mathrm{~N}$, and $6 \mathrm{~N}$ at end-stage. 
Could the absence of aggregation in $3 \mathrm{~N}$ be simply a function of reduced expression of G85R SOD1YFP in motor neurons of that cranial nerve relative to those in sites where aggregation occurs, for example, motor neurons of $12 \mathrm{~N}$ or spinal cord? We compared levels of mRNA encoding the mutant protein from laser captured motor neurons from $3 \mathrm{~N}, 12 \mathrm{~N}$, and spinal cord (SC) of a G85R SOD1YFP mouse (6 wk old, copy number 287) by qRT-PCR and observed that the amount of mRNA was reduced by $15-20 \%$ in $3 \mathrm{~N}$ relative to $12 \mathrm{~N}$ and spinal cord. Correspondingly, cytosolic YFP fluorescence intensity in $3 \mathrm{~N}$ motor neurons in perfusion-fixed tissue of two mice with similar copy number was also reduced by $25-30 \%$ relative to $12 \mathrm{~N}$ and spinal cord motor neurons lacking aggregates (see scatter plots of two "low copy" mice in Fig. 2).

Was a $25-30 \%$ reduction in the steady-state amount of G85R SOD1YFP, implied by the reduction in fluorescence, in $3 \mathrm{~N}$ relative to $12 \mathrm{~N}$ and spinal cord motor neurons sufficient to account for the lack of aggregation in 3N? To address this, we identified two G85R SOD1YFP mice with very high copy numbers, 341 and 329, respectively, exceeding by 25$50 \%$ the copy number of animals usually employed in our studies (i.e. ranging $240-280$, here termed "low copy"). We observed in these very high copy mice at 2 months of age that, corresponding with the copy number increase, the cytosolic fluorescence of their $3 \mathrm{~N}$ motor neurons was now increased by $\sim 20-50 \%$ ( $p<10^{-4}$; Fig. 2; compare blue plots mouse \#s $3 \& 4$ with blue plots \#s 1\&2). The fluorescence intensities in $3 \mathrm{~N}$ of the very high copy mice nearly completely overlapped within the fluorescence intensities in $12 \mathrm{~N}$ and SC of two age-matched low copy mice [compare blue scatter plots $(3 N)$ of mouse \#s $3 \& 4$ with red $(12 N)$ and green (SC) scatter plots of mouse \#s 1\&2]. The overlap of the fluorescence intensities was more formally addressed by assignment of a "positional overlapping score" (range 0.0-1.0) (5) to 
pairs of scatter plots of motor neuron intensities (see Tables 2 and 3). Table 2 shows pairwise comparisons of $3 \mathrm{~N}$ and $12 \mathrm{~N}$ sets from within the low copy and high copy mice and between them (sets shown in Fig. 2). Most relevant are the overlap percentage calculations (bold numbers in Table 2$)$ between low copy $3 \mathrm{~N}$ and low copy $12 \mathrm{~N}(8 \%, 26 \%, 27 \%, 43 \%)$ compared with the overlap percentages between high copy $3 \mathrm{~N}$ and low copy $12 \mathrm{~N}(48 \%, 55 \%$, $62 \%, 66 \%$ ). Clearly, the overlap of sets has been significantly enhanced by increased copy number. A similar increase in the positional overlapping scores are seen when $3 \mathrm{~N}$ of low copy and high copy are compared with spinal cord low copy (bold numbers in Table 3; $25 \%, 28 \%$, $43 \%, 52 \%$ vs $56 \%, 69 \%, 69 \%, 81 \%)$.

Strikingly, despite the florid aggregation in $12 \mathrm{~N}$ and in SC of the very high copy mice (resembling that shown in Fig. 1), not a single aggregate was found in sections comprising the entirety of $3 \mathrm{~N}$ from the two very high copy mice. Thus, when $3 \mathrm{~N}$ expression is increased as in the very high copy mice, reaching a level in most motor neurons that corresponds to that in aggregate-forming $12 \mathrm{~N}$ and SC motor neurons of low copy mice, there is still no aggregation observed. We thus conclude that $3 \mathrm{~N}$ motor neuron protection is not a function of the lower level of mutant protein expression in $3 \mathrm{~N}$ relative to $12 \mathrm{~N}$ and spinal cord.

We next addressed whether motor neuron survival in cranial nerve nuclei was affected by aggregation, comparing $3 \mathrm{~N}$ and $12 \mathrm{~N}$ from 3 mo control ChAT promoter-EGFP mice with G85R SOD1YFP mice at 3 months and end-stage, counting motor neurons in 20 micron coronal sections taken at 100 micron intervals down the length of these nuclei (totaling 5 sections for $3 \mathrm{~N}$ and 10 sections for $12 \mathrm{~N}$; see images in Fig. 3A and 3B for representative sections). The total of ChAT-positive motor neurons was then determined for each of 3 animals from the three groups. As shown in the bar graphs of Fig. 3A, the number of motor 
neurons in $3 \mathrm{~N}$ was indistinguishable when comparing ChAT-EGFP with G85R SOD1YFP 3 mo and endstage (the means were $297,290,327$, non-significant $p$ values with t-test). The lack of motor neuron loss in $3 \mathrm{~N}$ thus mirrors the lack of aggregation. By contrast, the mean motor neuron count for $12 \mathrm{~N}$ was reduced significantly in end-stage G85R SOD1YFP relative to ChAT-EGFP (Fig. 3B; 507 vs. 708, $p=0.01$ ). Thus for $12 \mathrm{~N}$, the occurrence of substantial aggregation was associated with significant cell loss, amounting to $\sim 30 \%$.

In sum, the foregoing data indicate that the cranial nerves supplying extraocular muscles in our SOD1-linked ALS mice are spared of aggregation relative to the other cranial motor nuclei, and where motor neuron survival was scored, there was no cell loss in $3 \mathrm{~N}$ (oculomotor) vs. significant cell loss in $12 \mathrm{~N}$ (hypoglossal) by end-stage. Thus, there appears to be a relationship between aggregation and cell loss, albeit it remains to be seen whether the former can drive the latter. It may rather be that the same cellular pathophysiology that drives aggregation can, at the same time, direct cell death. Regardless, the difference in behavior between $3 \mathrm{~N}$ and $12 \mathrm{~N}$ can serve as a basis for trying to understand mechanism(s) of protection/sensitivity. We presume that ultimately other mouse models of ALS will likely exhibit the same extraocular motor sparing and that, more generally, at least some of the features of motor collapse in SOD1-linked ALS mice can serve to provide understanding of "sporadic" versions of ALS where, potentially, at least some similar steps of motor neuron loss are occurring. 
Acknowledgments

We thank HHMI for supporting this work. E.V.T. was supported by the Nelson Fund.

We are especially grateful to Elizabeth Engle, Children's Hospital/Harvard Medical School, for suggesting inspection of cranial nuclei in G85R SOD1YFP mice for aggregates.

\section{References}

1. Cwik, V.A., ALS clinical motor signs and symptoms, in Amyotrophic Lateral Sclerosis, Mitsumoto, Przedborski, Gordon (eds), 2006, Taylor and Francis, chapter 5, p.111.

2. Wang, J., Farr, G.W., Zeiss, C.J., Rodriguez-Gil, D.J., Wilson, J.H., et al. (2009).

Progressive aggregation despite chaperone associations of a mutant SOD1-YFP in transgenic mice with ALS. Proc. Natl. Acad. Sci. USA 106, 1392-1397.

3. Hadzipasic, M., Tahvildari, B., Nagy, M., Bian, M., Horwich, A.L., and McCormick, D.A. (2014). Selective degeneration of a physiological subtype of spinal motor neuron in mice with SOD1-linked ALS. Proc. Natl. Acad. Sci. USA 111, 16883-16888.

4. Nagy, M., Fenton, W.A., Li, D., Furtak, K., and Horwich, A.L. (2016). Extended survival of misfolded G85R SOD1-linked ALS mice by transgenic expression of chaperone Hsp110. Proc. Natl. Acad. Sci. USA 113, 5424-5428.

5. Mahmoud, O., Harrison, A., Perperoglou, A., Gul, A., Khan, Z., Metodiev, M.V., and Lausen, B. (2014). A feature selection method for classification within functional genomics experiments based on the proportional overlapping score. BMC Bioinformatics 15, 274. 
bioRxiv preprint doi: https://doi.org/10.1101/304857; this version posted October 24,2018 . The copyright holder for this preprint (which was not certified by peer review) is the author/funder, who has granted bioRxiv a license to display the preprint in perpetuity. It is made available under aCC-BY-NC-ND 4.0 International license.

6. Thomas, E.V., Fenton, W.A., McGrath, J., and Horwich, A.L. (2017) Transfer of pathogenic and non-pathogenic cytosolic proteins between spinal cord motor neurons in vivo in chimeric mice. Proc. Natl. Acad. Sci. USA 114(15):E3139-E3148. 
A. $3 \mathrm{mo}$ G85R SOD1YFP

Fig. 1
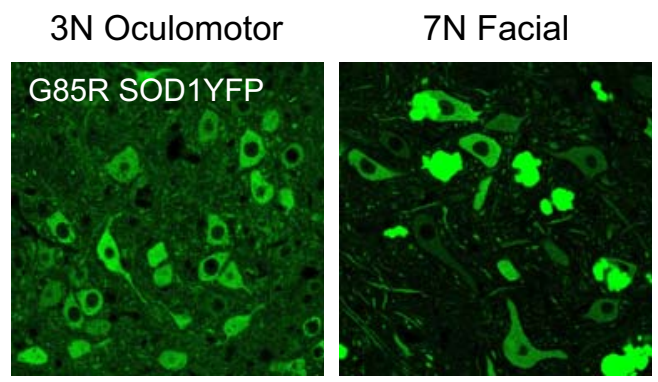

12N Hypoglossal
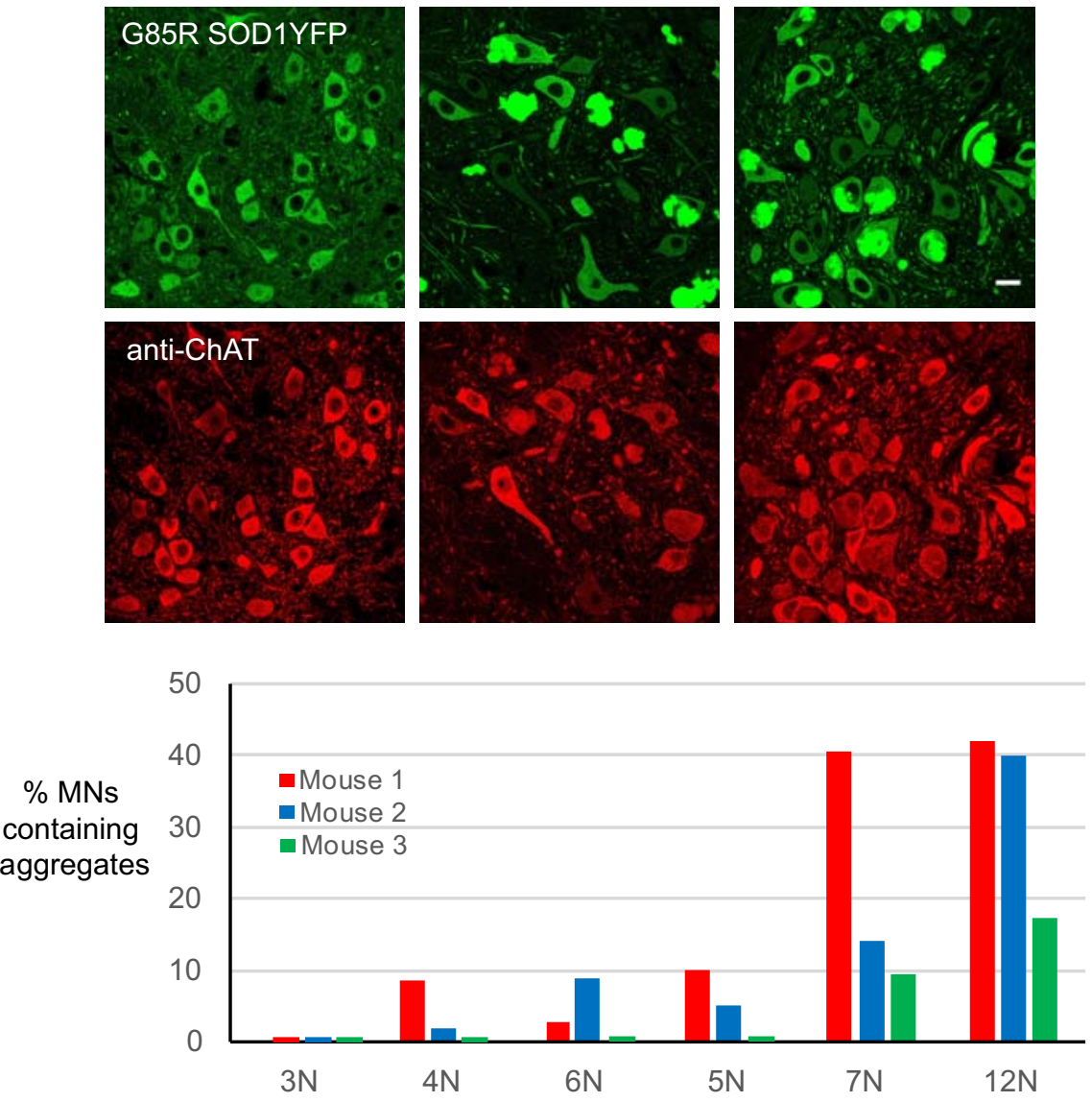

B. End-stage G85R SOD1YFP
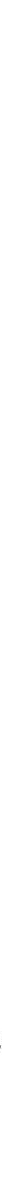

3N Oculomotor Nucleus 4N Trochlear Nucleus $6 \mathrm{~N}$ Abducens Nucleus $5 \mathrm{~N}$ Trigeminal Nucleus $7 \mathrm{~N}$ Facial Nucleus $12 \mathrm{~N}$ Hypoglossal Nucleus

$$
\text { (1) }
$$

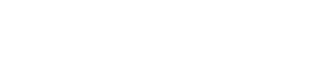


Fig. 1. Aggregation of SOD1YFP in cranial nerve motor nuclei.

Panels $A$ and $B$ show aggregation in ChAT positive motor neurons from cranial nerve nuclei of G85R SOD1YFP mice at 3 months (panel A) and end-stage (panel B), with representative images shown (top) from $3 \mathrm{~N}$ (oculomotor), $7 \mathrm{~N}$ (facial), and 12N (hypoglossal), and bar graphs for $3 \mathrm{~N}, 4 \mathrm{~N}, 6 \mathrm{~N}$ (extraocular innervation) and $5 \mathrm{~N}, 7 \mathrm{~N}, 12 \mathrm{~N}$ (other motor nuclei, excluding the motor division of $10 \mathrm{~N}$, which did exhibit aggregates). Scale bars are 20 microns and apply to all images. Aggregates are more abundant in the latter group at both 3 months and endstage, but $5 \mathrm{~N}$ is less affected and exceeds the extraocular nuclei only at end-stage. Sample preparation, ChAT immunostaining, and microscopy were carried out as in ref. 6 . Aggregates were counted in 20 micron coronal sections from 3 mice at 3 months and 3 mice at end-stage. The neuron counts used to generate the bar graphs are shown in Table 1. 


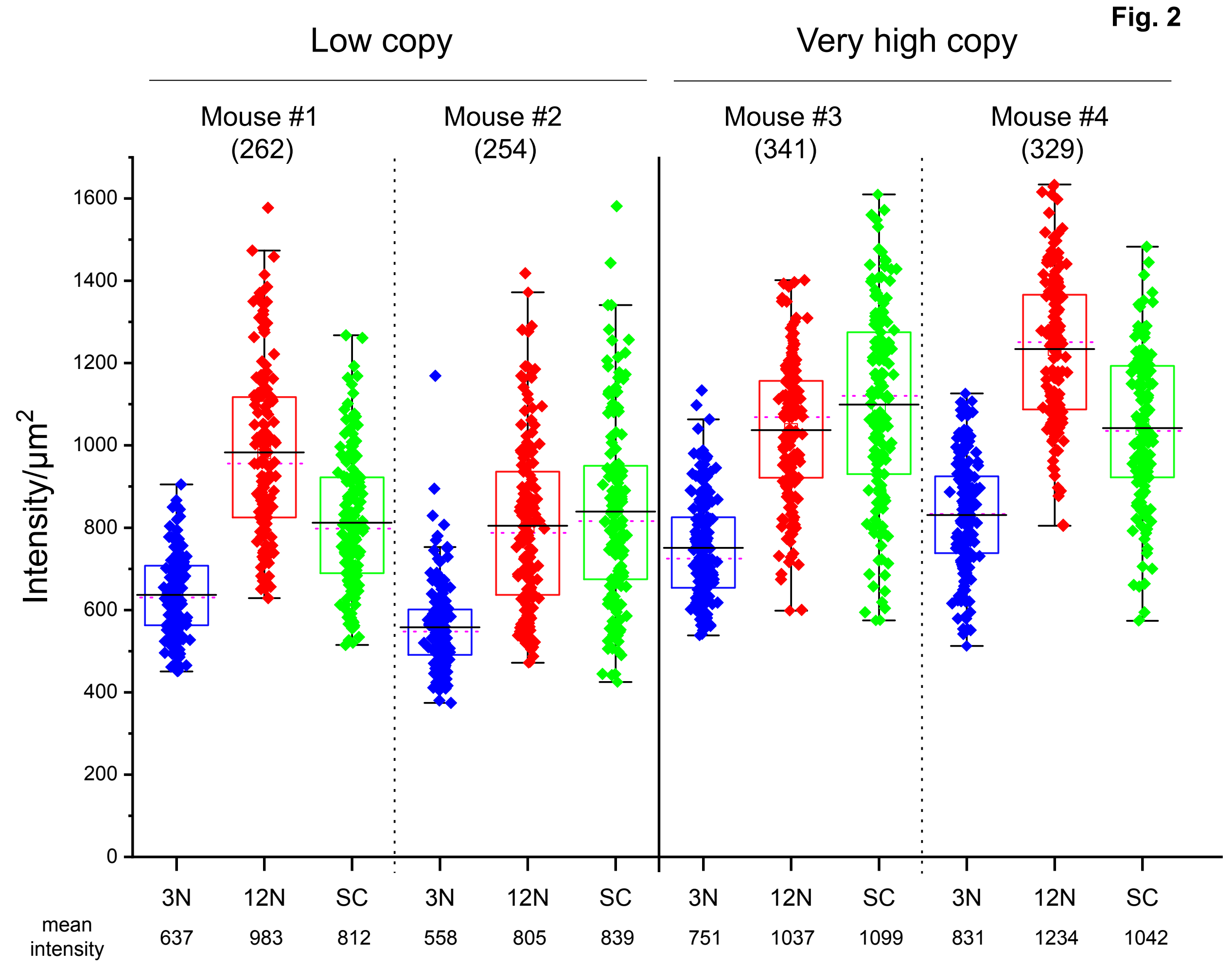


Fig. 2. Cytosolic YFP fluorescence intensity of motor neurons from cranial nerves $3 \mathrm{~N}$ and $12 \mathrm{~N}$ and from spinal cord from low and very high copy number G85R SOD1YFP transgenic mice. Scatter plots show YFP fluorescence intensities of 150 motor neurons each from oculomotor $(3 \mathrm{~N})$ and hypoglossal $(12 \mathrm{~N})$ cranial nerve motor nuclei and spinal cord (SC) of two month old G85R SOD1YFP transgenic mice with "low" and very high copy number, mouse \#1,2 and 3,4, respectively. For each tissue, 150 motor neurons (devoid of aggregates in the case of $12 \mathrm{~N}$ and SC) were analyzed. A $20 \mu \mathrm{m}^{2}$ region of interest was randomly chosen from $1 \mu \mathrm{m}$ thick optical (z) sections, and gray scale values were summed for the pixels in that area. The scatter plots encompass all 150 neurons, presented as individual points. The boxes indicate $75^{\text {th }}$ (top) and $25^{\text {th }}$ (bottom) percentiles, the means are indicated at bottom below the tissue designation, and the whiskers (horizontal black lines) indicate 1.5 SD above and below the mean. 
A.

Oculomotor Nucleus (3N)
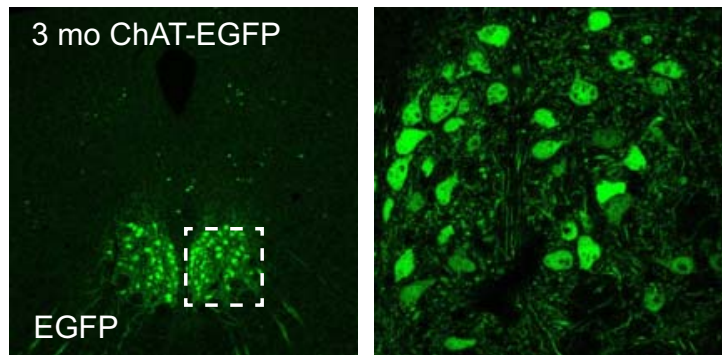

3 mo G85R SOD1YFP

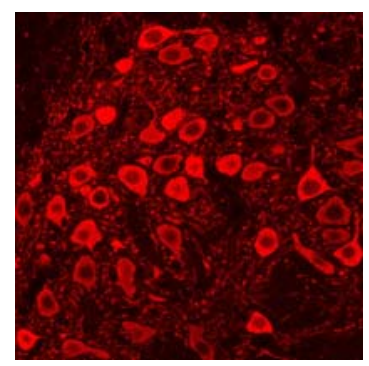

anti-ChAT

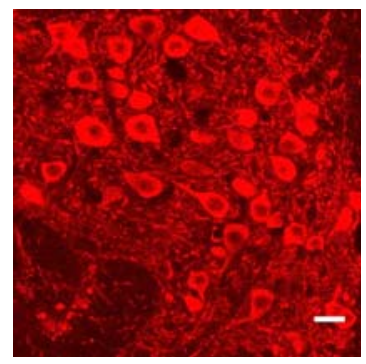

anti-ChAT

B.

Hypoglossal Nucleus (12N)

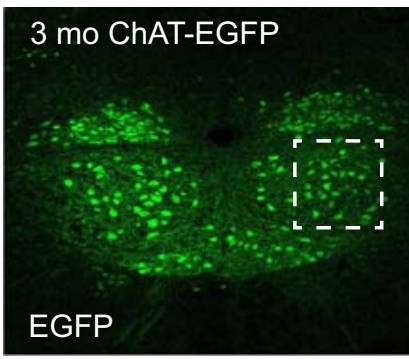

\section{3 mo G85R SOD1YFP}

\section{anti-ChAT}

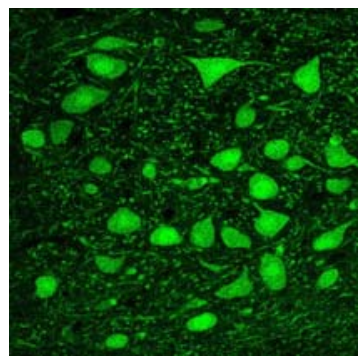

End- stage G85R SOD1YFP$$
r-5
$$$$
1
$$$$
1=1
$$

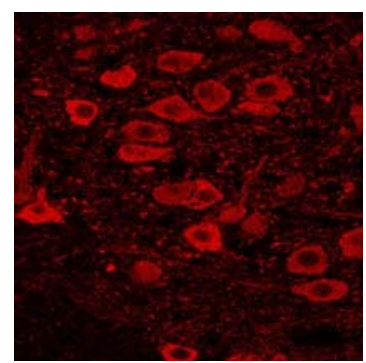

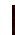

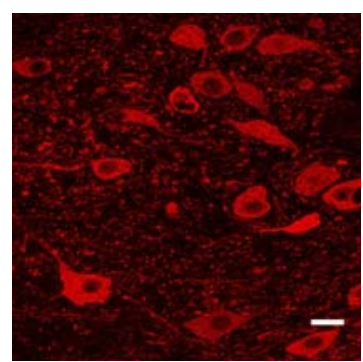

Motor
Neuron
Number

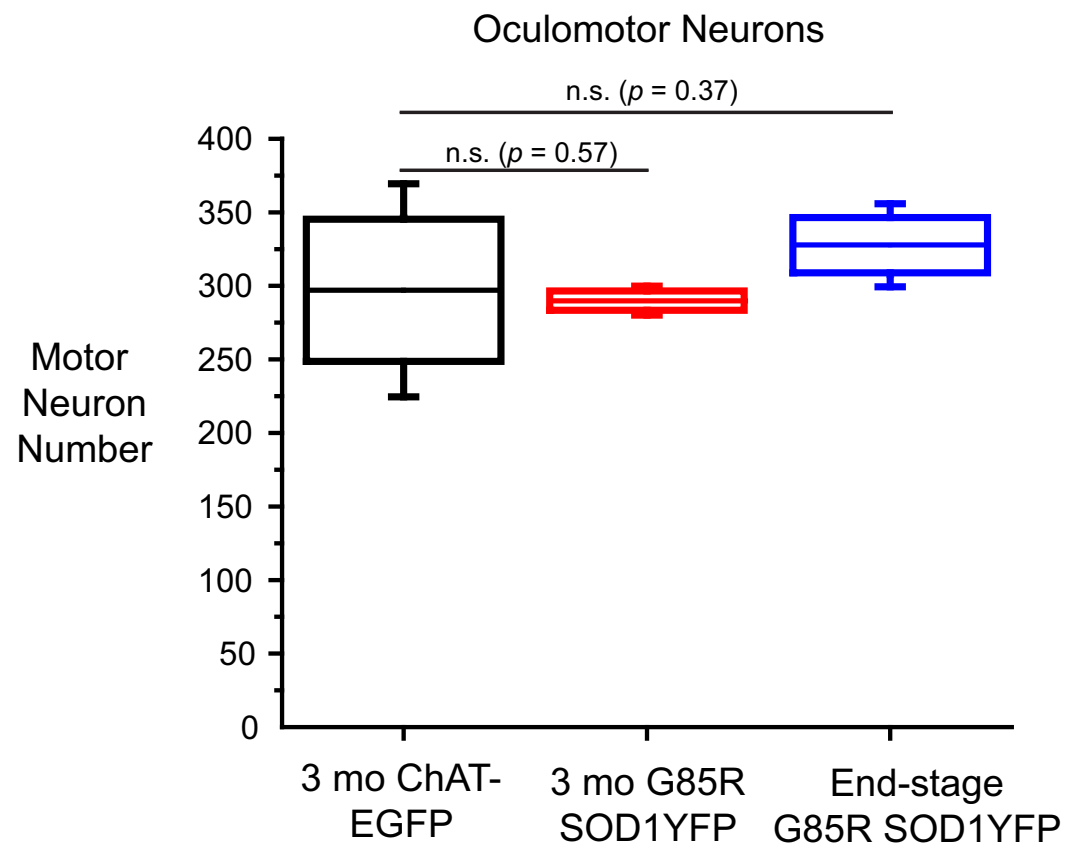

Motor

Neuron

Number

Fig. 3

Hypoglossal Motor Neurons

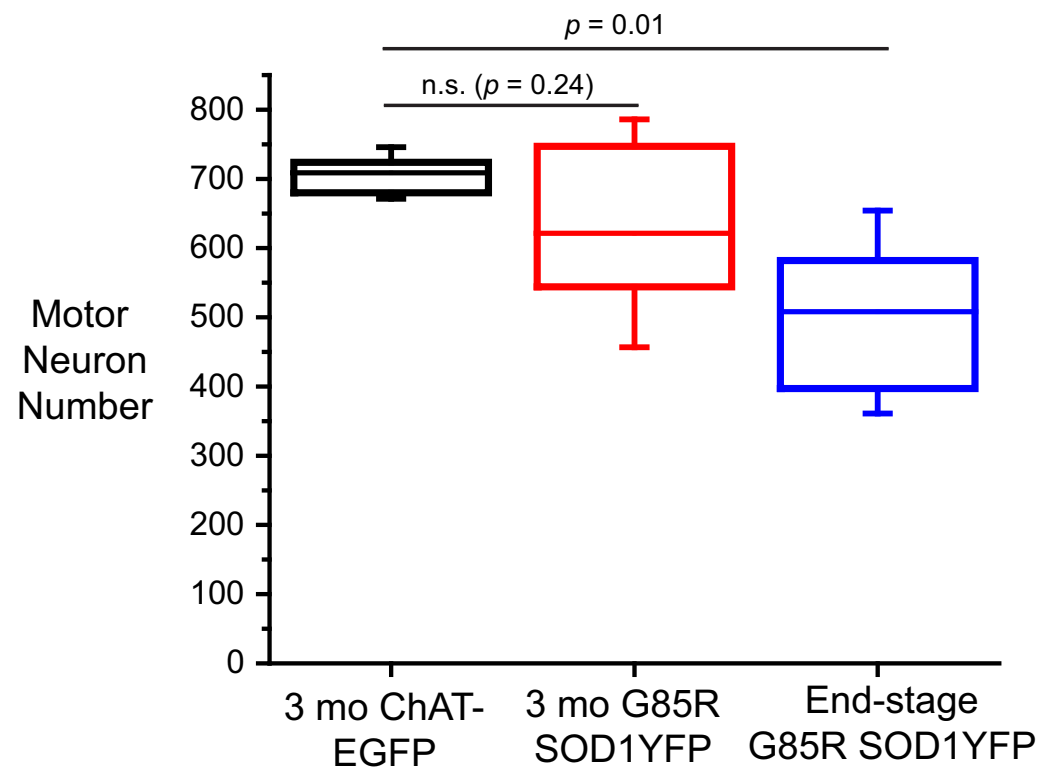


Fig. 3. Survival of motor neurons in cranial nerve nuclei 3 and 12 (oculomotor and hypoglossal) of G85R SOD1YFP ALS mice.

Panels $\mathrm{A}$ and $\mathrm{B}$ show ChAT positive motor neurons of $3 \mathrm{~N}$ and $12 \mathrm{~N}$, respectively, determined either from direct GFP fluorescence of ChAT-EGFP mice or anti-ChAT antibody staining of sections from G85R SOD1YFP mice of 3 months or end-stage. At left are low magnification images (scale bar 200 microns) and at right are high resolution images of the indicated areas (scale bar 20 microns). At right are box plots of the motor neuron counts corresponding to the respective mice ( $n=3$ for each designation). The upper and lower extents of the boxes indicate the $75^{\text {th }}$ and $25^{\text {th }}$ percentiles of the data, respectively. The lines inside the boxes indicate the means, and the whiskers \pm 1.5 S.D. $p$ values determined by paired t-tests (Origin); n.s., non-significant.

ChAT positive motor neurons were counted in 20 micron (coronal) sections taken every 100 microns along the length of the respective nuclei ( 5 such sections from $3 \mathrm{~N}$ and 10 from $12 \mathrm{~N}$ ). The sections were stained with anti-ChAT antibody (6), positive cells counted, and counts summed across the sections for the cranial nucleus of a given animal. The numbers were compared with those in the corresponding cranial nuclei of a ChAT promoter-EGFP mouse. 3 mice each of ChAT-EGFP (3 mo), 3 mo G85R SOD1YFP, and end-stage G85R SOD1YFP were analyzed. 
Table 1. Percentage of motor neurons with aggregates in cranial nerve nuclei of G85R SOD1YFP mice at 3 months of age and at end-stage.

\begin{tabular}{|c|c|c|c|c|c|c|}
\hline $\begin{array}{c}\text { 3 mo G85R } \\
\text { SOD1YFP }\end{array}$ & $\mathbf{3 N}$ & $\mathbf{4 N}$ & $\mathbf{6 N}$ & $\mathbf{5 N}$ & $\mathbf{7 N}$ & $\mathbf{1 2 N}$ \\
\hline \hline Mouse 1 (cn 298) & $0 \%(0 / 79)$ & $8 \%(5 / 59)$ & $3 \%(1 / 36)$ & $10 \%(6 / 60)$ & $41 \%(48 / 118)$ & $42 \%(47 / 112)$ \\
\hline Mouse 2 (cn 247) & $0 \%(0 / 76)$ & $2 \%(1 / 43)$ & $9 \%(1 / 11)$ & $5 \%(4 / 78)$ & $14 \%(32 / 226)$ & $40 \%(44 / 109)$ \\
\hline Mouse 3 (cn 290) & $0 \%(0 / 70)$ & $0 \%(0 / 44)$ & $0 \%(0 / 25)$ & $0 \%(0 / 124)$ & $10 \%(24 / 251)$ & $17 \%(17 / 98)$ \\
\hline
\end{tabular}

\begin{tabular}{|c|c|c|c|c|c|c|}
\hline $\begin{array}{c}\text { End stage G85R } \\
\text { SOD1YFP }\end{array}$ & 3N & $\mathbf{4 N}$ & $\mathbf{6 N}$ & $\mathbf{5 N}$ & $\mathbf{7 N}$ & $\mathbf{1 2 N}$ \\
\hline \hline Mouse 1 (cn 262) & $0 \%(0 / 92)$ & $0 \%(0 / 54)$ & $0 \%(0 / 75)$ & $9 \%(6 / 69)$ & $30 \%(41 / 138)$ & $34 \%(34 / 99)$ \\
\hline Mouse 2 (cn 269) & $0 \%(0 / 93)$ & $4 \%(2 / 54)$ & $2 \%(1 / 67)$ & $7 \%(6 / 84)$ & $19 \%(27 / 143)$ & $37 \%(37 / 101)$ \\
\hline Mouse 3 (cn 268) & $0 \%(0 / 90)$ & $0 \%(0 / 22)$ & $0 \%(0 / 62)$ & $5 \%(1 / 22)$ & $22 \%(38 / 175)$ & $32 \%(32 / 99)$ \\
\hline
\end{tabular}

cn = copy number of G85R SOD1YFP transgene. 
Table 2. Proportional overlapping scores comparing motor neuron G85R SODYFP intensities between cranial nerve nuclei in low copy and very high copy mice.

\begin{tabular}{|c|c|c|c|c|c|c|c|c|}
\hline & $1-3 \mathrm{~N}$ & $1-12 \mathrm{~N}$ & $2-3 N$ & $2-12 N$ & $3-3 N$ & $3-12 N$ & $4-3 N$ & $4-12 \mathrm{~N}$ \\
\hline $1-3 N$ & & $\begin{array}{l}\mathbf{0 . 2 7} \\
(0.20,0.33)\end{array}$ & $\begin{array}{l}0.65 \\
(0.58,0.70)\end{array}$ & $\begin{array}{l}\mathbf{0 . 4 3} \\
(0.34,0.57)\end{array}$ & $\begin{array}{l}0.68 \\
(0.60,0.73)\end{array}$ & $\begin{array}{l}0.12 \\
(0.06,0.18)\end{array}$ & $\begin{array}{l}0.47 \\
(0.38,0.53)\end{array}$ & $\begin{array}{l}0.01 \\
(0.00,0.02)\end{array}$ \\
\hline $1-12 \mathrm{~N}$ & & & $\begin{array}{l}\mathbf{0 . 0 8} \\
(0.04,0.13)\end{array}$ & $\begin{array}{l}0.71 \\
(0.60,0.79)\end{array}$ & $\begin{array}{l}\mathbf{0 . 4 8} \\
(0.37,0.60)\end{array}$ & $\begin{array}{l}0.84 \\
(0.68,0.93)\end{array}$ & $\begin{array}{l}\mathbf{0 . 6 2} \\
(0.49,0.71)\end{array}$ & $\begin{array}{l}0.61 \\
(0.54,0.67)\end{array}$ \\
\hline $2-3 N$ & & & & $\begin{array}{l}\mathbf{0 . 2 6} \\
(0.18,0.36)\end{array}$ & $\begin{array}{l}0.37 \\
(0.28,0.43) \\
\end{array}$ & $\begin{array}{l}0.02 \\
(0.00,0.05)\end{array}$ & $\begin{array}{l}0.19 \\
(0.10,0.24)\end{array}$ & $\begin{array}{l}0 \\
(0,0)\end{array}$ \\
\hline $2-12 N$ & & & & & $\begin{array}{l}0.55 \\
(0.44,0.80) \\
\end{array}$ & $\begin{array}{l}0.58 \\
(0.44,0.69)\end{array}$ & $\begin{array}{l}\mathbf{0 . 6 6} \\
(0.51,0.85)\end{array}$ & $\begin{array}{l}0.32 \\
(0.21,0.42)\end{array}$ \\
\hline $3-3 N$ & & & & & & $\begin{array}{l}0.33 \\
(0.23,0.47)\end{array}$ & $\begin{array}{l}0.76 \\
(0.68,0.85)\end{array}$ & $\begin{array}{l}0.10 \\
(0.05,0.19)\end{array}$ \\
\hline $3-12 N$ & & & & & & & $\begin{array}{l}0.57 \\
(0.45,0.63)\end{array}$ & $\begin{array}{l}0.67 \\
(0.60,0.72)\end{array}$ \\
\hline $4-3 N$ & & & & & & & & $\begin{array}{l}0.24 \\
(0.17,0.31)\end{array}$ \\
\hline $4-12 \mathrm{~N}$ & & & & & & & & \\
\hline
\end{tabular}

Data from Fig. 2 were analyzed by the algorithms in ref. 5. For each pair, the proportional overlapping score is shown, followed by the $95 \%$ confidence interval in parentheses. The overlaps highlighted in bold indicate comparisons discussed in the text. 
Table 3. Proportional overlapping scores comparing motor neuron G85R SODYFP intensities between spinal cord and cranial nerve nuclei in low copy and very high copy mice.

\begin{tabular}{|l|c|c|}
\hline & $1-S C$ & $2-S C$ \\
\hline $1-3 N$ & $\mathbf{0 . 5 2}(0.38,0.58)$ & $\mathbf{0 . 4 3}(0.30,0.51)$ \\
\hline $1-12 \mathrm{~N}$ & $0.68(0.60,0.78)$ & $0.74(0.67,0.83)$ \\
\hline $2-3 \mathrm{~N}$ & $\mathbf{0 . 2 8}(0.19,0.34)$ & $\mathbf{0 . 2 5}(0.15,0.31)$ \\
\hline $2-12 \mathrm{~N}$ & $0.81(0.64,0.95)$ & $0.94(0.69,0.96)$ \\
\hline $3-3 \mathrm{~N}$ & $\mathbf{0 . 6 9}(0.54,0.87)$ & $\mathbf{0 . 5 6}(0.41,0.76)$ \\
\hline $3-12 \mathrm{~N}$ & $0.58(0.47,0.68)$ & $0.61(0.50,0.71)$ \\
\hline $4-3 \mathrm{~N}$ & $\mathbf{0 . 8 1}(0.62,0.94)$ & $\mathbf{0 . 6 9}(0.49,0.83)$ \\
\hline $4-12 \mathrm{~N}$ & $0.29(0.20,0.39)$ & $0.33(0.27,0.49)$ \\
\hline
\end{tabular}

Data from Fig. 2 were analyzed by the algorithms in ref. 5. For each pair, the proportional overlapping score is shown, followed by the $95 \%$ confidence interval in parentheses. The overlaps highlighted in bold indicate comparisons discussed in the text. 